\title{
MicroRNA-509-3p inhibits cancer cell proliferation and migration by targeting the mitogen-activated protein kinase kinase kinase 8 oncogene in renal cell carcinoma
}

\author{
ZHENGMING SU ${ }^{1-3}$, DUQUN CHEN ${ }^{1,4}$, ENPU ZHANG ${ }^{1,2}$, YIFAN LI ${ }^{1,4}$, ZUHU YU $^{1,4}$, MIN SHI $^{1,2}$, \\ ZHIMAO JIANG ${ }^{1,2}$, LIANGCHAO NI ${ }^{1,2}$, SHANGQI YANG ${ }^{1,2}$, YAOTING GUI ${ }^{1,2}$, \\ JIONGXIAN YE $^{1-3}$ and YONGQING LAI ${ }^{1-3}$
}

\author{
${ }^{1}$ Department of Urology, Peking University Shenzhen Hospital, Shenzhen, Guangdong 518036; \\ ${ }^{2}$ Guangdong and Shenzhen Key Laboratory of Male Reproductive Medicine and Genetics, Institute of Urology, \\ Peking University Shenzhen Hospital, Shenzhen PKU-HKUST Medical Center, Shenzhen, Guangdong 518036; \\ ${ }^{3}$ Department of Urology, Shantou University Medical College, Shantou, Guangdong 515041; \\ ${ }^{4}$ Department of Urology, Anhui Medical University, Hefei, Anhui 230032, P.R. China
}

Received May 15, 2014; Accepted February 10, 2015

DOI: $10.3892 / \mathrm{mmr} .2015 .3498$

\begin{abstract}
RNAs (miRNAs; miR) are a class of small non-coding RNA molecules, which are involved in the pathogenesis of human diseases through the negative regulation of gene expression. Previous studies have demonstrated that miR-509-3p is a novel miRNA associated with cell proliferation and migration in 786-O renal cell carcinoma (RCC) cells. However, the mechanism of action of miR-509-3p in RCC remains to be elucidated. The present study aimed to examine the functional role and mechanism of miR-509-3p in the development of RCC. The results demonstrated that the expression levels of miR-509-3p were downregulated in the 786-O and ACHN RCC cell lines compared with the normal tissues of 10 patients with RCC, as determined by reverse transcription-quantitative polymerase chain reaction. The mRNA expression levels of mitogen-activated protein kinase kinase kinase 8 (MAP3K8) were upregulated in the RCC cell lines. Functional investigations demonstrated that the overexpression of miR-509-3p inhibited the migration and proliferation of the RCC cells, as determined by wound scratch and 3-(4,5-dimethylthiazol-2-yl)-2,5-diphenyltetrazolium bromide assays. Luciferase reporter assays revealed that the overexpression of miR-509-3p reduced the transcriptional activity of MAP3K8. Furthermore, the present study
\end{abstract}

Correspondence to: Professor Yongqing Lai or Professor Jiongxian Ye, Department of Urology, Peking University Shenzhen Hospital, 1120 Lianhua Road, Shenzhen, Guangdong 518036, P.R. China

E-mail: yqlord@163.com

E-mail: yjx66@126.com

Key words: renal cell carcinoma, microRNA-509-3p, mitogenactivated protein kinase kinase kinase 8 demonstrated that the ectopic transfection of miR-509-3p led to a significant reduction in the mRNA and protein expression levels of MAP3K8 in the RCC cells. Finally, knockdown of MAP3K8 inhibited the migration and proliferation of the RCC cells. Therefore, the results of the present study demonstrated that the miR-509-3p RCC suppressor was a significant regulator of the MAP3K8 oncogene, suggesting that it may have a potential therapeutic role in the treatment of RCC.

\section{Introduction}

Renal cell carcinoma (RCC) is the most common neoplasm of the kidney, with $\sim 80 \%$ of patients with RCC diagnosed worldwide with the clear cell RCC subtype (1). It has been reported that the prognosis of advanced RCC is poor, with a 5-year survival rate of 5-10\% (2,3). At diagnosis, $30 \%$ of patients with RCC exhibit metastatic disease, and radical nephrectomy remains the primary treatment option for patients with RCC, due to resistance of the tumors to radiation and chemotherapy $(4,5)$. Although several environmental and genetic factors have been found to be associated with RCC, the molecular mechanisms underlying the initiation and progression of RCC remain to be fully elucidated (6). Therefore, there is an urgent requirement for the identification of sensitive and reliable biomarkers for the development of novel targeted therapies for RCC.

MicroRNA (miRNA), are small non-coding RNA of 20-22 nucleotides, which regulate gene expression by targeting messenger RNA (mRNA) through translational repression or mRNA degradation (7). To date, $\sim 2,000$ human miRNAs have been identified, which are involved in the regulation of biological processes, including proliferation, apoptosis, migration and differentiation (8). Several miRNAs have been implicated as oncogenes or as tumor suppressors, and are aberrantly expressed in various types of cancer, including RCC (9-12). Previous studies have demonstrated, through transfection with pre-miR-509-3p in vitro, that miR-509-3p is downregulated in 
$\mathrm{RCC}$, which is associated with cell proliferation and migration in 786-O RCC cells. However, the mechanism of action of miR-509-3p in RCC remains to be elucidated. Therefore, there is a requirement to further investigate the functional significance of miR-509-3p, and to identify novel targets regulated by miR-509-3p in 786-O and ACHN RCC cells.

Mitogen-activated protein kinase kinase kinase 8 (MAP3K8), also termed COT, is an oncogene encoding a member of the serine/threonine protein kinase family (13). The encoded protein localizes to the cytoplasm and activates the MAP kinase and c-Jun N-terminal kinase (JNK) kinase pathways (13). Previous studies have demonstrated that overexpression of MAP3K8 correlates with the development of breast cancer (14), prostate cancer (15) and endometrioid carcinoma (16). In addition, increased expression of MAP3K8 is a significant prognostic factor of poor survival rates in patients with colorectal cancer (CRC), which suggests that MAP3K8 is important in carcinogenesis (17). Bioinformatics has revealed that the 3'UTR of MAP3K8 contains a putative binding site for miR-509-3p. However, the regulation of miR-509-3p in RCC, and its association with MAP3K8, have not been reported.

In the present study, the expression pattern of miR-509-3p in RCC cells was examined, and the effects of miR-509-3p on the proliferation and migration of two RCC cell lines were assessed. To improve understanding of the underlying regulatory mechanism, a target MAP3K8 gene was identified using a luciferase reporter assay in the RCC cell lines. The present study aimed to establish whether overexpression of miR-509-3p affected the mRNA and protein expression levels of MAP3K8, and whether knockdown of MAP3K8 affected the migration and proliferation of the RCC cells.

\section{Materials and methods}

Tissue samples. The present study was approved by the Institutional Review Board and Ethical Committee of Peking University Shenzhen Hospital (Shenzhen, China). All patients involved provided written informed consent. A total of 10 normal specimens were obtained from 10 patients diagnosed with RCC following nephrectomy at Peking University Shenzhen Hospital in 2013. The normal specimens were located $2.0 \mathrm{~cm}$ away from visible RCC lesions. All tissue samples were reviewed and classified with hematoxylin \& eosin staining (Beijing Disinbio Science \& Technology Co., Ltd., Beijing, China), and the disease Tumor Node Metastasis stages of the patients were classified according to the 2009 American Joint Committee on Cancer staging system (18). The clinical and pathological characteristics of the 10 patients with RCC included in the present study are shown in Table I. The fresh tissue samples were immediately immersed in RNAlater (Qiagen, Hilden, Germany), following surgical resection, were stored at $4^{\circ} \mathrm{C}$ overnight and were subsequently frozen in liquid nitrogen for storage at $-80^{\circ} \mathrm{C}$ until analysis. A pathologist confirmed that all the specimens were derived from normal tissues.

Cell culture and cell transfection. The 786-O and ACHN cell lines used in the present study were obtained from the American Type Culture Collection (Manassas, VA, USA. These cell lines were maintained in Dulbecco's modified Eagle's medium
(DMEM; Invitrogen Life Technologies, Carlsbad, CA, USA), supplemented with $10 \%$ fetal bovine serum (FBS; Shanghai ExCell Biology, Inc., Shanghai, China) and 1\% antibiotics (100 U/ml penicillin and $100 \mathrm{mg} / \mathrm{ml}$ streptomycin sulfates; BioWiseTech Co., Ltd., Shanghai, China) and were cultured in a humidified air atmosphere of $5 \% \mathrm{CO}_{2}$ at $37^{\circ} \mathrm{C}$.

Small interfering (si)RNA (siRNA-MAP3K8; si-MAP3K8) were designed to target MAP3K8. The miR-509-3p mimics, si-MAP3K8 and negative control were transfected into the 786-O and ACHN human RCC cells lines. The sequences are listed in Table II. The RNA oligoribonucleotides were purchased from GenePharma (Shanghai, China). Prior to transfection, $3 \times 10^{5}$ cells were seeded into six-well plates and cultured for $24 \mathrm{~h}$ at $37^{\circ} \mathrm{C}$. Once the cells had reached $60-80 \%$ confluence, they were transfected with RNA using Lipofectamine $2000{ }^{\circledR}$ reagent (Invitrogen Life Technologies), according to the manufacturer's instructions.

RNA extraction and reverse transcription-quantitative polymerase chain reaction $R T-q P C R$ analysis. The total RNA was extracted from cells and tissues using TRIzol ${ }^{\circledR}$ reagent (Invitrogen Life Technologies), according to the manufacturer's instructions. RT-qPCR analysis was performed to detect the expression levels of miR-509-3p in the 10 human normal renal tissues and the 786-O and ACHN RCC cell lines. A total of $1 \mu \mathrm{g}$ total RNA was reverse transcribed into cDNA using a miScript Reverse Transcription kit (Qiagen), according to the manufacturer's instructions. qPCR was performed using a SYBR green assay (Takara Bio, Inc., Dalian, China) on a Roche LightCycler 480 machine (Roche Applied Science, Mannheim, Germany), according to the manufacturer's instructions and using U6 as an internal control. The $20 \mu 1$ reaction mixture contained $10 \mu \mathrm{l}$ 2X QuantiTect SYBR Green PCR Master mix, $2 \mu 1$ 10X miScript Universal Primer, $0.4 \mu 1$ specific microRNA primer, $1 \mu \mathrm{l}$ cDNA template and RNase-free water. The primers used for miR-509-3p and U6 are listed in Table II, and were purchased from Promega Corporation (Madison, WI, USA). The program for PCR was: $95^{\circ} \mathrm{C}$ for $15 \mathrm{~min}$, followed by 40 cycles at $94^{\circ} \mathrm{C}$ for $15 \mathrm{sec}, 55^{\circ} \mathrm{C}$ for $30 \mathrm{sec}$ and $72^{\circ} \mathrm{C}$ for $30 \mathrm{sec}$.

To determine the mRNA expression levels of MAP3K8, RT was performed using Revert Aid First Strand cDNA synthesis kit (Thermo Fisher Scientific, Vilnius, Lithuania), according to the manufacturer's instructions. The $20 \mu 1$ PCR mixture contained $10 \mu 1$ 2X QuantiTect SYBR Green PCR Master mix (Takara Bio, Inc.), $1 \mu \mathrm{l}$ cDNA template, $1 \mu \mathrm{l}$ each primer and RNase-free water. The program for PCR was: $95^{\circ} \mathrm{C}$ for $15 \mathrm{~min}$, followed by 45 cycles of $94^{\circ} \mathrm{C}$ for $15 \mathrm{sec}, 57^{\circ} \mathrm{C}$ for $30 \mathrm{sec}$ and $72^{\circ} \mathrm{C}$ for $30 \mathrm{sec}$. The expression levels of MAP3K8 were evaluated using the comparative threshold cycle $(\mathrm{Ct})$ method (9). GAPDH was used as an internal control. The primers used for MAP3K8 and GAPDH are listed in Table II. All reactions were performed in triplicate.

Cell migration assay. Cell migration was examined using a wound scratch assay, as described previously (9). The 786-O and ACHN cells $(\sim 500,000$ each) were seeded into 6-well plates and, following $24 \mathrm{~h}$ incubation at $37^{\circ} \mathrm{C}$, were transfected with the miRNA-509-3p mimic, si-MAP3K8 or negative control using Lipofectamine 2000 (Invitrogen Life Technologies). 
Table I. Clinical characteristics of the patients with ccRCC.

\begin{tabular}{|c|c|c|c|c|}
\hline Patient & Gender & Age (years) & TNM & Histological type \\
\hline 1 & M & 32 & T1N0M0 & ccRCC \\
\hline 2 & M & 36 & T2NOM0 & ccRCC \\
\hline 3 & M & 41 & T2N0M0 & $\mathrm{ccRCC}$ \\
\hline 4 & M & 40 & T1N0M0 & $\mathrm{ccRCC}$ \\
\hline 5 & M & 27 & T1N0M0 & $\mathrm{ccRCC}$ \\
\hline 6 & $\mathrm{~F}$ & 52 & T2NOM0 & ccRCC \\
\hline 7 & $\mathrm{~F}$ & 40 & T1N0M0 & ccRCC \\
\hline 8 & M & 58 & T2NOM0 & $\mathrm{ccRCC}$ \\
\hline 9 & $\mathrm{~F}$ & 48 & T2N0M0 & $\mathrm{ccRCC}$ \\
\hline 10 & M & 52 & T4NOMO & $\mathrm{ccRCC}$ \\
\hline
\end{tabular}

ccRCC, clear cell renal cell carcinoma; TNM, Tumor Node Metastasis staging.

Table II. Primer sequences used in the present study.

\begin{tabular}{|c|c|c|}
\hline Name & Primer & Sequence $\left(5^{\prime}-3^{\prime}\right)$ \\
\hline \multirow[t]{2}{*}{ miR-509-3p mimics } & Forward & TGATTGGTACGTCTGTGGGTAG \\
\hline & Reverse & ACCCACAGACGTACCAATCATT \\
\hline \multirow[t]{2}{*}{ si-MAP3K8 } & Forward & GCGCCTTTGGAAAGGTATATT \\
\hline & Reverse & TATACCTTTCCAAAGGCGCTT \\
\hline \multirow[t]{2}{*}{ Negative control } & Forward & TTCTCCGAACGTGTCACGTTT \\
\hline & Reverse & ACGTGACACGTTCGGAGATT \\
\hline \multirow[t]{2}{*}{ miR-509-3p } & Forward & TGATTGGTACGTCTGTGGGTAG \\
\hline & Reverse & Universal primers (miScript SYBR Green PCR kit) \\
\hline \multirow[t]{2}{*}{ U6 } & Forward & CTCGCTTCGGCAGCACA \\
\hline & Reverse & ACGCTTCACGAATTTGCGT \\
\hline \multirow[t]{2}{*}{ MAP3K8 } & Forward & ATGGAGTACATGAGCACTGGA \\
\hline & Reverse & GCTGGCTCTTCACTTGCATAAAG \\
\hline \multirow[t]{2}{*}{ GAPDH } & Forward & GGAGCGAGATCCCTCCAAAAT \\
\hline & Reverse & GGCTGTTGTCATACTTCTCATGG \\
\hline \multirow[t]{2}{*}{ MAP3K8-3'UTR-WT } & Forward & CCGCTCGAGTACCAATCACAAGGATAATGC \\
\hline & Reverse & ATTTGCGGCCGCATTATCCTTGTGATTGGTA \\
\hline \multirow[t]{2}{*}{ MAP3K8-3'UTR-MUT } & Forward & CCGCTCGAGGCAACCGACCAAGGATAATGC \\
\hline & Reverse & ATTTGCGGCCGCATTATCCTTGGTCGGTTGC \\
\hline
\end{tabular}

miR, microRNA; si, small interfering; MAP3K8, mitogen-activated protein kinase kinase kinase 8; UTR, untranslated region; WT, wild-type; MUT, mutant; PCR, polymerase chain reaction.

Following $6 \mathrm{~h}$ incubation at $37^{\circ} \mathrm{C}$, a sterile $200 \mu \mathrm{l}$ pipette tip and marker was used to create a scratch in the cell monolayer. The cells were then washed with phosphate-buffered saline (PBS) three times and incubated at $37^{\circ} \mathrm{C}$. Images of the scratches were captured with a digital camera (DMIRB; Leica Microsystems GmbH, Wetzlar, Germany) 0 and $24 \mathrm{~h}$ after the scratches were made. The MIAS-2000 software program (Leica Microsystems $\mathrm{GmbH}$ ) was used to determine the distance of migration. The experiments were performed in three independent repeats in triplicate and were analyzed in a blinded-manner by at least two observers.
Cell proliferation assay. The cell proliferation was determined using a 3-(4,5-dimethylthiazol-2-yl)-2,5-diphenyltetrazolium bromide (MTT) assay, according to the manufacturer's instructions. The 786-O and ACHN cells were seeded into 96-well culture plates at a cell density of 6,000 cells/well in growth medium (DMEM containing 10\% FBS) and transfected with 10 pmol miRNA-509-3p mimic, si-MAP3K8 or negative control. The cell growth was assessed by adding $20 \mu 1$ MTT (5 mg/ml; Sigma-Aldrich, St. Louis, MO, USA) to each well, and incubating at $37^{\circ} \mathrm{C}$ for $4 \mathrm{~h}$. The proliferation assay was performed for 3 days and cell growth was examined every 
$24 \mathrm{~h}$. The reaction was inhibited by the addition of $150 \mu \mathrm{l}$ dimethyl sulfloxide (DMSO; Sigma-Alrdich). Following agitation for $15 \mathrm{~min}$ at room temperature, the optical density (OD) of each sample, at a wavelength of $490 \mathrm{~nm}$, was measured using an Enzyme Immunoassay instrument (Model 680; Bio-Rad Laboratories, Inc., Hercules, CA, USA). All assays were performed in triplicate.

Target gene prediction. Prediction of the miRNA target was performed using computational algorithms, according to the base-pairing rules between the miRNA and mRNA target sites, location of binding sequences within the target' 3'UTR, and conservation of target binding sequences within associated genomes. In the present study, genes were predicted using the miRWalk (http://www.umm.uni-heidelberg. de/apps/zmf/mirwalk), TargetScanv5.1 (http://www.targetscan. org), microRNA (http://www.microrna.org/microrna/home. do) and miRDB (http://mirdb.org/miRDB/index.html) computational algorithms.

Luciferase reporter assay. The present study generated luciferase reporter plasmids containing the MAP3K8 3'UTR fragment and the miRNAs target sequences inserted between the XhoI-NotI restriction sites in the 3'UTR of the hRluc gene in the psiCHECK-2 luciferase vector (Promega Corporation). To generate the psiCHECK-2-3'-UTR-WT plasmid, primer sequences for the 3'UTR of MAP3K8 mRNA were designed. In order to confirm the binding sites of the miR-509-3p, two short fragments of the MAP3K8 3'UTR were synthesized, which contained the potential binding sites and the matching sequences (underlined in Table II). The potential binding sites were mutated manually by exchanging the $\mathrm{G}$ and $\mathrm{T}$, A and C, to produce the psiCHECK-2-3'-UTR-MUT plasmid. The forward and reverse sequences are shown in Table II (MAP3K8-3'UTR-WT and MAP3K8-3'UTR-MUT'). These short fragments were cloned into the psiCHECK-2 luciferase vector and the constructs were verified by bidirectional sequencing between the XhoI-NotI restriction sites in the 3'UTR of the hRluc gene in the psiCHECK-2 luciferase vector. Sequencing was performed by Invitrogen Life Technologies. The luciferase reporter construct, together with the miR-509-3p mimics or negative control, were subsequently transfected into the 786-O and ACHN cells using Lipofectamine 2000 (Invitrogen Life Technologies). At $24 \mathrm{~h}$ post-transfection, the firefly and Renilla luciferase activities of the cells were detected using a Dual-Luciferase ${ }^{\circledR}\left(D L^{\mathrm{TM}}\right)$ Reporter Assay system (Promega Corporation) on the Modulus ${ }^{\mathrm{TM}}$ Single Tube Multimode Reader (Bio-Systems International, Beloit, WI, USA). Normalized data were calculated as the quotient of Renilla/firefly luciferase activities. These experiments were performed in duplicate and were repeated at least three times.

Protein extraction and western blot analysis. The proteins were extracted for western blot analysis $48 \mathrm{~h}$ after transfecting the 786-O and ACHN cells with the miRNA-509-3p mimics, si-MAP3K8 and negative control. The cells were washed three times with PBS and lysed in radioimmunoprecipitation buffer (Sigma-Aldrich) to extract the total cellular protein. The protein content was determined using an Enhanced BCA protein assay kit (Beyotime Institute of Biotechnology,
Shanghai, China). The total protein $(30 \mu \mathrm{g})$ from each sample was separated by electrophoresis on $10 \%$ sodium dodecyl sulfate-polyacrylamide gels prior to being transferred onto polyvinylidene fluoride membranes (Millipore, Billerica, MA, USA) for $2 \mathrm{~h}$. The membranes were blocked for $1 \mathrm{~h}$ at room temperature using 5\% non-fat milk and subsequently incubated in Tris-buffered saline with $0.05 \%$ Tween- $20^{\circledR}$ (TBST; Ameresco, Solon, OH, USA), containing rabbit polyclonal immunoglobulin (Ig)G anti-MAP3K8 (cat. no. 152104; 1:500; Abcam, Hong Kong, China) or rabbit polyclonal $\beta$-actin (cat. no. 600-532; 1:5,000; Novus, Littleton, CO, USA) antibodies overnight at $4^{\circ} \mathrm{C}$. After $24 \mathrm{~h}$, the membranes were washed with PBS three times and incubated with peroxidase-conjugated goat anti-rabbit Ig G secondary antibody (cat. no. 7074; 1:5,000; Cell Signaling Technology, Inc., Danvers, MA, USA) at $37^{\circ} \mathrm{C}$ for $2 \mathrm{~h}$. The protein expression was evaluated using chemiluminescence [Immun-Star ${ }^{\mathrm{TM}}$ Horseradish Peroxidase (HRP) Chemiluminescence kit; Bio-Rad Laboratories, Inc.] and exposure to Kodak film (Kodak, Rochester, NY, USA).

Statistical analysis. The data are expressed as the mean \pm standard deviation from at least three separate experiments. Statistical significance was determined using Student's t-test. $\mathrm{P}<0.05$ was considered to indicate a statistically significant difference. Statistical analyses was performed using SPSS 16.0 statistical software (SPSS, Inc., Chicago, IL, USA).

\section{Results}

miR-509-3p is downregulated and MAP3K8 is upregulated in RCC cell lines. To evaluate the mRNA expression levels of miR-509-3p and MAP3K8 in the 786-O and ACHN RCC cell lines, equal quantities of total RNA from the normal adjacent tissues of 10 patients with RCC were pooled together as a control. The mRNA expression levels of miR-509-3p and MAP3K8 in each RCC cell line were compared with the pool of 10 normal specimens by RT-qPCR. The results demonstrated that all the cell lines downregulated the expression of miR-509-3p (Fig. 1A) and upregulated MAP3K8 (Fig. 1B), suggesting that miR-509-3p acted as a tumor suppressor and MAP3K8 acted as an oncogene in the RCC cells.

Proliferative and migratory abilities of RCC cells are regulated by miR-509-3p. To further confirm the role of miR-509-3p in RCC cells, scratch and MTT assays were performed to evaluate the proliferative and migratory capacities of the 786-O and ACHN cells treated with the miR-509-3p mimics or negative control for $24 \mathrm{~h}$. As shown in Fig. 2A, cell migration was significantly inhibited in the groups transfected with miR-509-3p compared with those in the negative control group. The inhibitory rates of migration were $31.68 \%$ in the $786-\mathrm{O}$ cells $(\mathrm{P}<0.05)$ and $28.66 \%$ in the ACHN cells $(\mathrm{P}<0.05)$, indicating that miR-509-3p had a negative effect on cellular migration. The MTT assay demonstrated that the relative cell proliferation in the miR-509-3p transfectants was significantly decreased by 4.32 (24 h; $\mathrm{P}<0.05), 12.14$ (48 h; $\mathrm{P}<0.05)$ and $16.79 \%(72 \mathrm{~h} ; \mathrm{P}<0.05)$ in the $786-\mathrm{O}$ cells. Similarly, the proliferation of the ACHN cells was inhibited by $5.83(24 \mathrm{~h}, \mathrm{P}<0.05)$, 12.1 (48 h; $\mathrm{P}<0.05)$ and 14.39 (72 h; $\mathrm{P}<0.05)$. The MTT assay results are shown in Fig. $2 \mathrm{~B}$. These results demonstrated that 
A

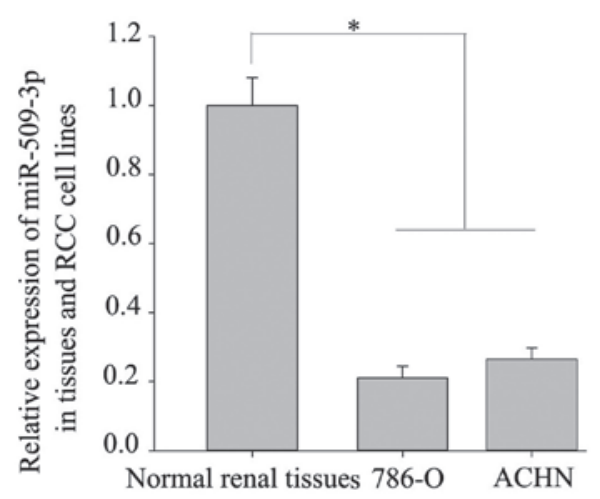

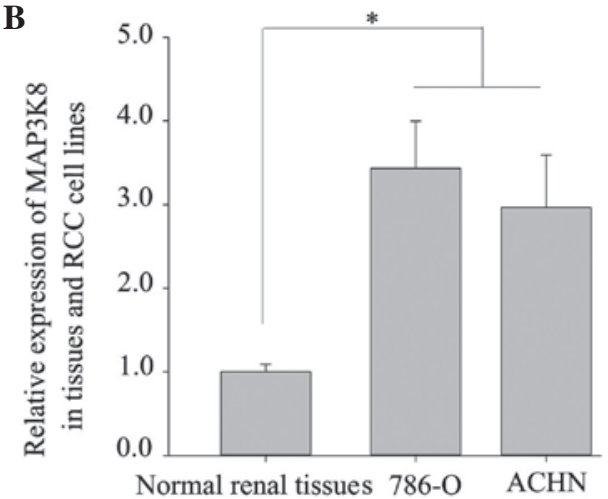

Figure 1. Relative mRNA expression levels of (A) miR-509-3p and (B) MAP3K8 in the 786-O and ACHN RCC cell lines and normal renal tissues, measured by reverse transcription-quantitative polymerase chain reaction. The results demonstrated that miR-509-3p was downregulated and MAP3K8 was upregulated in the RCC cell lines compared with the normal renal tissue. All reactions were performed in triplicate and data are expressed as the mean \pm standard deviation. (" $\mathrm{P}<0.05$, compared with the normal tissues). RCC, renal cell carcinoma; miR, microRNA; MAP3K8, mitogen-activated protein kinase kinase kinase 8 .

A
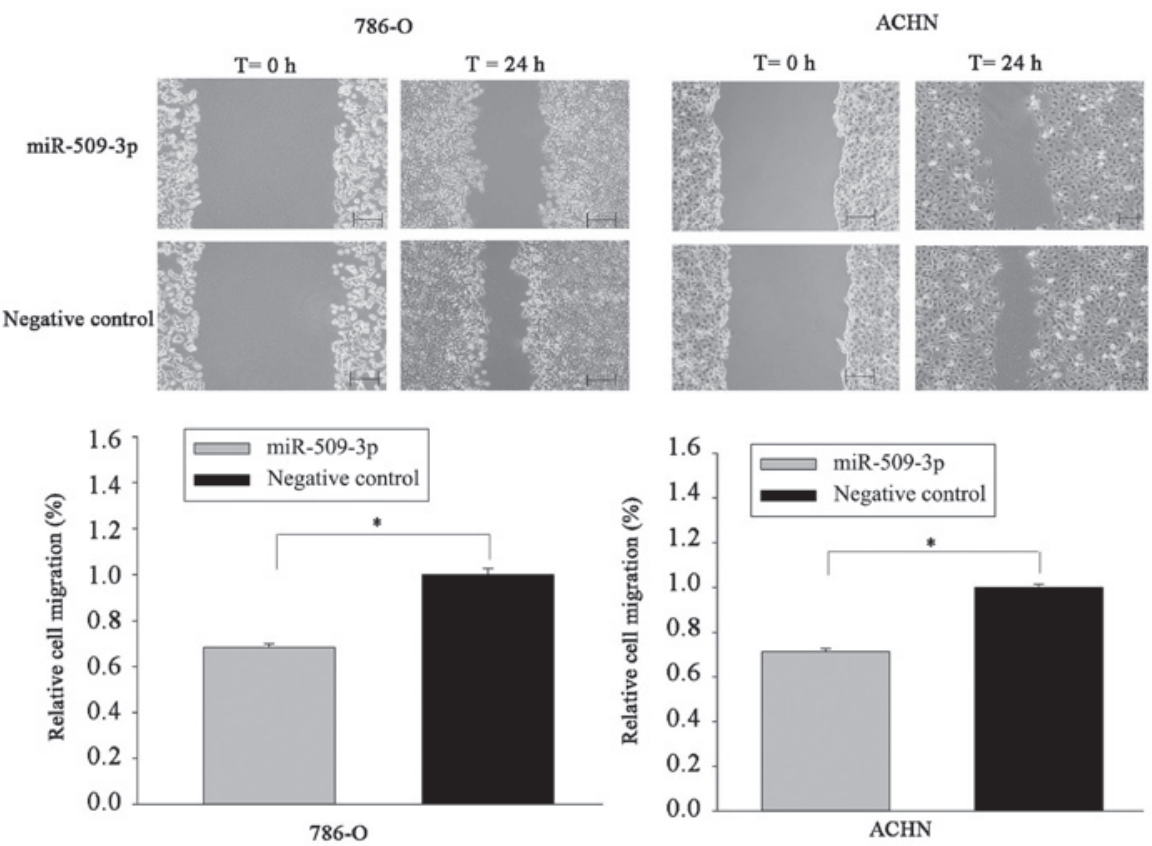

B
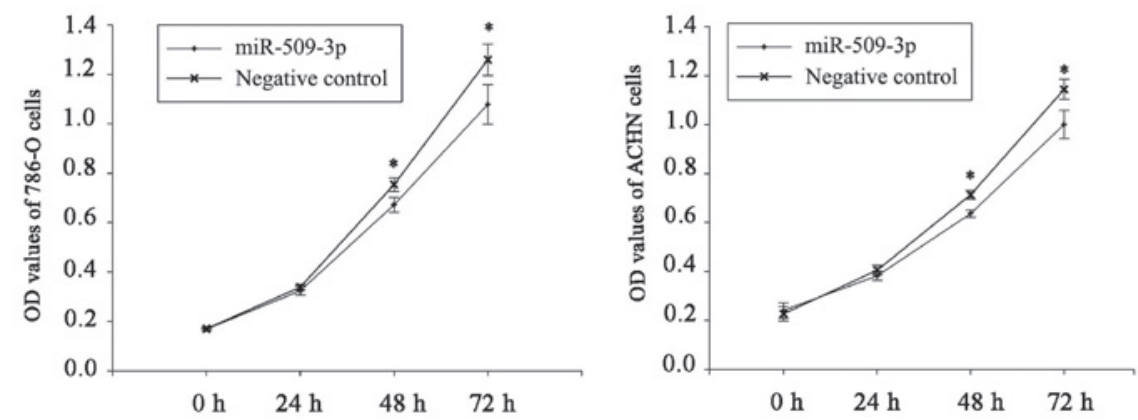

Figure 2. Effects of miR-509-3p on cell migration and proliferation in the RCC cell lines. (A) miR-509-3p inhibited migration in 786-O and ACHN cells (Scale bar, $200 \mu \mathrm{m}$ ). (B) miR-509-3p inhibited proliferation in 786-O and ACHN cells. All experiments were performed three times and a representative image is shown. Data are expressed as the mean \pm standard deviation. ("P<0.05, compared with the negative control). RCC, renal cell carcinoma; OD, optical density; miR, microRNA.

miR-509-3p markedly affected the migration and proliferation of the RCC cell lines.

MAP3K8 is the target gene of miR-509-3p. To predict the targets of miR-509-3p, four computational algorithms
(miRWalk, TargetScan, microrna and miRDB) were used. From the potential targets, the present study focused on the oncogene, MAP3K8. As shown in the Fig. 3A, MAP3K8 revealed putative target sites at position 2397-2419 of the 3'UTR, with an exact match in the seed region at position 
A

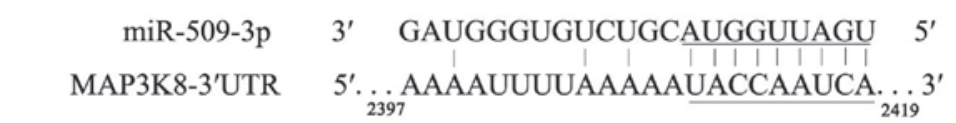

MAP3K8-3'UTR-WT

5'... CCGCTCGAGTACCAATCACAAGGATAATGC. . . 3'

MAP3K8-3'UTR-MUT $\quad 5^{\prime} \ldots$ CCGCTCGAGGCAACCGACCAAGGATAATGC. . . 3'
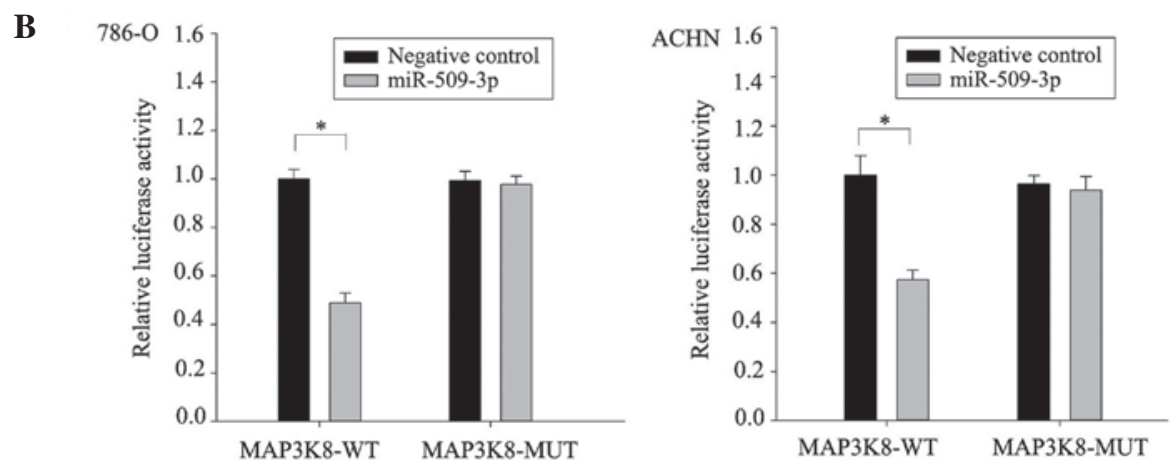

Figure 3. MAP3K8 is the target gene of miR-509-3p. (A) Putative duplex formation between miR-509-3p and the MAP3K8 3'UTR. The seed-recognizing sites are underlined. A 21-nucleotide-long fragment of the MAP3K8 3'UTR was synthesized, containing WT potential binding sites. The MUT fragment was generated by exchanging the $\mathrm{G}$ and $\mathrm{T}, \mathrm{A}$ and $\mathrm{C}$ on the putative binding sites. (B) Luciferase reporter constructs containing the 3'UTR of MAP3K8, together with the miR-509-3p mimics or negative control, were transfected into the 786-O and ACHN cells. After $48 \mathrm{~h}$, luciferase activity was detected. The relative luciferase activity of the reporter, containing the WT 3'UTR was significantly suppressed when miR-509-3p was cotransfected into the 786-O and ACHN cells. By contrast, the luciferase activity of the MUT reporter was unaffected by cotransfection with miR-509-3p. The experiments were performed in duplicate and repeated three times. Data are expressed as the mean \pm standard deviation $\left({ }^{*} \mathrm{P}<0.05\right.$, compared with the negative control). RCC, renal cell carcinoma; miR, microRNA; MAP3K8, mitogen-activated protein kinase kinase kinase 8; UTR, untranslated region; WT, wild-type; MUT, mutant.

A

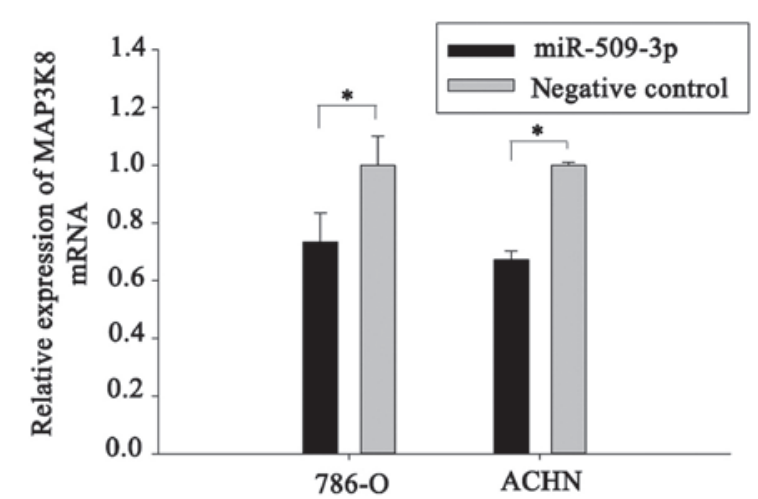

B

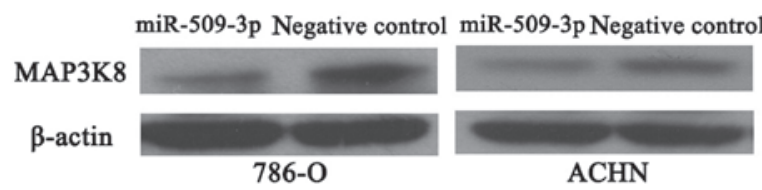

Figure 4. (A) Cells transfected with miR-509-3p mimics exhibited decreased mRNA expression levels of MAP3K8 compared with those transfected with the negative control. (B) Overexpression of miR-509-3p caused a decrease in the protein expression levels of MAP3K8 in the two renal cell carcinoma cell lines. All experiments were performed three times and a representative image is shown Data are expressed as the mean \pm standard deviation. ("P $<0.05$, compared with the negative control). miR, microRNA; MAP3K8, mitogen-activated protein kinase kinase kinase 8.

2410-2419. This site is located in conserved regions of the MAP3K8 3'UTR in Homo sapiens (microRNA; http://www. microrna.org/microrna/home.do). To confirm that MAP3K8 was a target of miR-509-3p, a luciferase reporter assay was performed. The relative luciferase activity of the reporter, which contained the wild-type 3'UTR, was significantly suppressed when the miR-509-3p was cotransfected in the 786-O and ACHN cells (Fig. 3B). By contrast, the luciferase activity of the mutant reporter was unaffected following cotransfection with miR-509-3p (Fig. 3B). Collectively, these results demonstrated that MAP3K8 was a target gene of miR-509-3p and identified the site of interaction in the 3'UTR of MAP3K8.

MAP3K8 is downregulated by miR-509-3p in the RCC cell lines. The RT-qPCR and western blot analyses were performed to confirm whether the expression of miR-509-3p affected the expression of endogenous MAP3K8 at the transcriptional and translational levels. Consistent with the results from the luciferase report assay, the cells transfected with the 
A

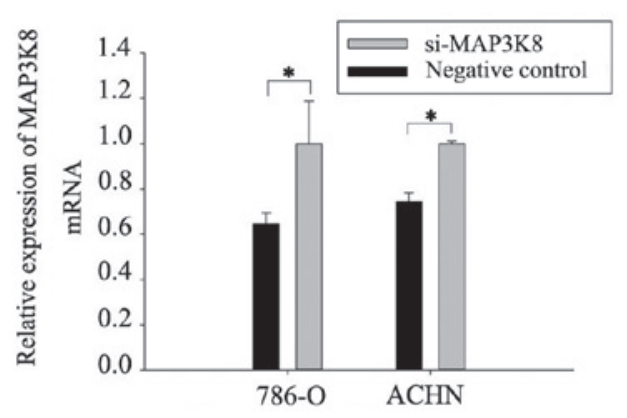

C
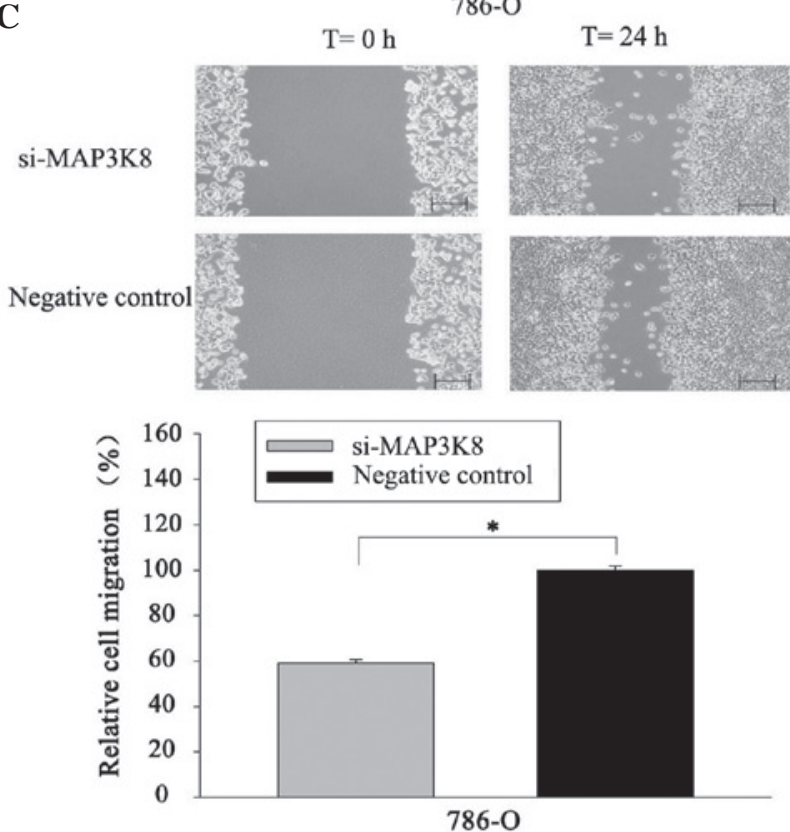

D

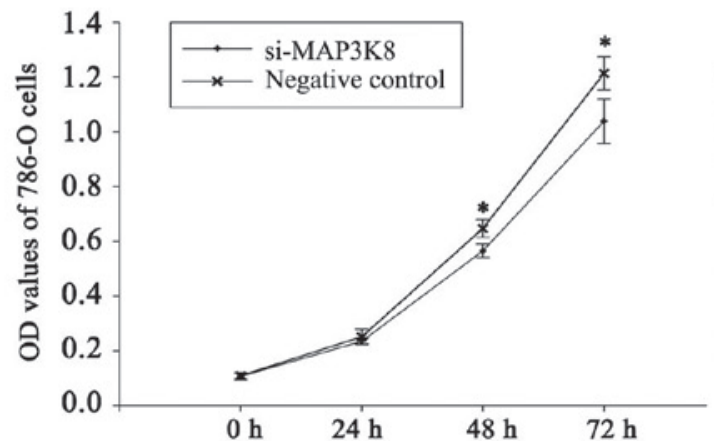

B

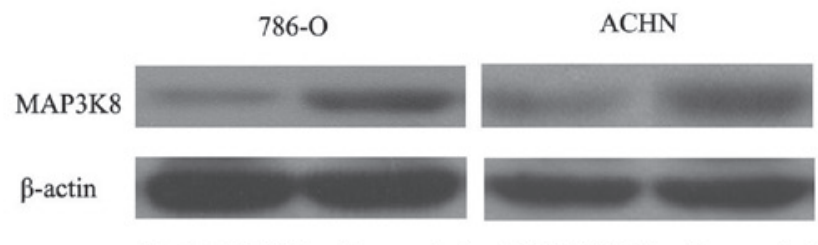

si-MAP3K8 Negative control si-MAP3K8 Negative control

ACHN

$\mathrm{T}=0 \mathrm{~h} \quad \mathrm{~T}=24 \mathrm{~h}$
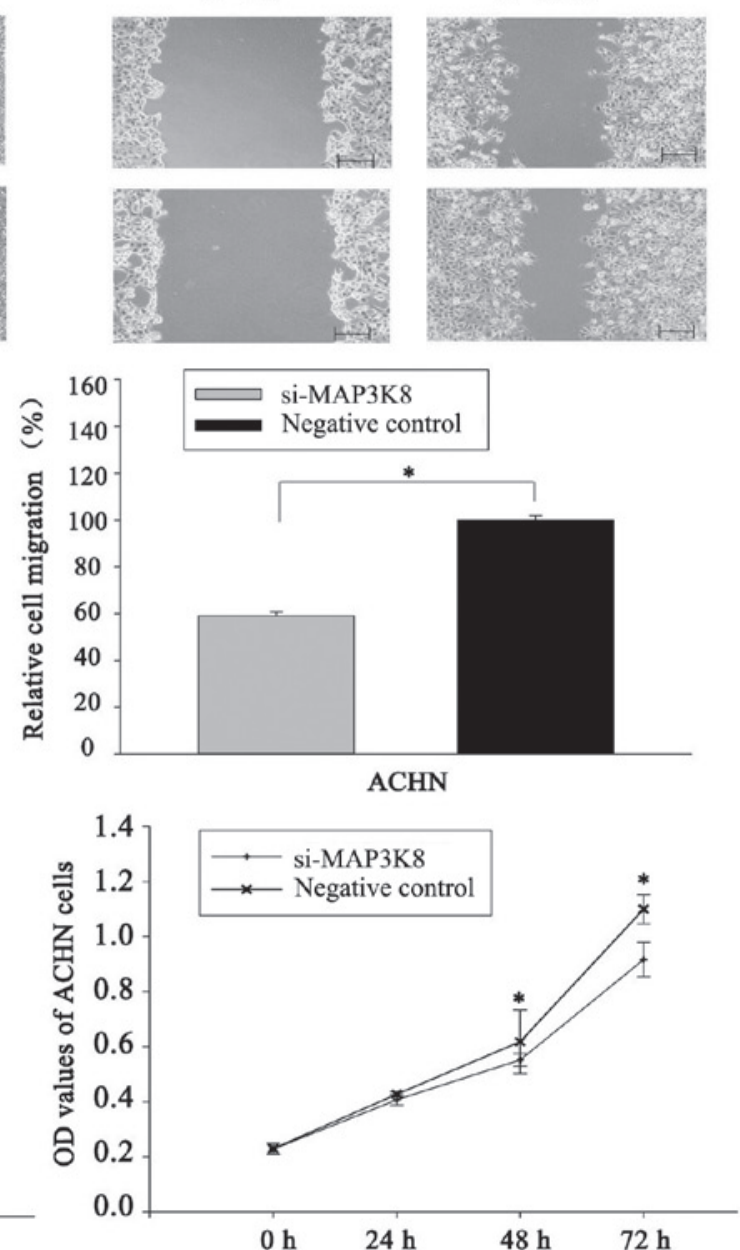

Figure 5. Silencing of MAP3K8 in the 786-O and ACHN RCC cell lines using si-MAP3K8. The (A) mRNA and (B) protein expression levels of MAP3K8 were markedly repressed following si-MAP3K8 transfection. (C) Migration of the 786-O and ACHN cells were significantly inhibited in the groups transfected with si-MAP3K8 compared with those in the negative control (Scale bar, $200 \mu \mathrm{m}$ ). (D) si-MAP3K8 suppressed the growth of the 786-O and ACHN cells compared with the negative control. All experiments were performed three times and a representative image is shown. Data are expressed as the mean \pm standard deviation (" $\mathrm{P}<0.05$, compared with the negative control). RCC, renal cell carcinoma; si, small interfering; MAP3K8, mitogen-activated protein kinase kinase kinase 8 .

miR-509-3p mimics exhibited decreased mRNA expression of MAP3K8 (Fig. 4A) compared with those transfected with the negative control. The overexpression of miR-509-3p caused a decrease in the protein expression levels of MAP3K8 in the two RCC cell lines (Fig. 4B).

Effects of si-MAP3K8 transfection on the RCC cells. To examine the significance of MAP3K activity in the cell variability of RCC cells, loss-of-function analyses were performed in the 786-O and ACHN cells transfected with the si-MAP3K8 sequences. The mRNA and protein expression levels of MAP3K8 were markedly repressed following si-MAP3K8 transfection (Fig. 5A and B).

The scratch assays were performed to observe the importance of MAP3K8 in cell migration. As shown in Fig. 5C, cell migration was significantly inhibited in the groups transfected with si-MAP3K8 compared with those in the negative control group. The inhibitory rates of migration were $26.6 \%$ in the 
786-O cells $(\mathrm{P}<0.05)$ and $40.92 \%$ in the ACHN cells $(\mathrm{P}<0.05)$, indicating that knockdown of MAP3K8 inhibited the migration of the RCC cells.

To determine the potential role of MAP3K8 on the proliferation of RCC cells, MTT assays were performed. The si-MAP3K8 group and negative control group were measured at $0,24,48$ and $72 \mathrm{~h}$ following transfection. The OD values demonstrated that the proliferation of 786-O cells decreased by $6.88,14.51$ and $16.8 \%(\mathrm{P}<0.05)$, while the proliferation rates of the ACHN cells decreased by 4.91, 12.01 and $19.95 \%(\mathrm{P}<0.05)$ at 24,48 and $72 \mathrm{~h}$ after transfection, respectively. This suggested that silencing of MAP3K8 suppressed the growth of the 786-O and ACHN cells compared with the negative control (Fig. 5D).

\section{Discussion}

RCC is a highly vascularized tumor, which accounts for $3 \%$ of all malignancies in adults and has the highest rate of mortality of all urological malignancies $(19,20)$. However, the current treatment options of surgery, radiation and chemotherapy are relatively ineffective in treating advanced RCC. Therefore, it is vital to understand the molecular mechanisms underlying the metastasis of RCC and to identify alternative treatment strategies.

Previous studies have demonstrated that miRNAs exhibit unique expression profiles in different types of cancer at different stages, and are important in the initiation and progression of several diseases. This suggests that miRNAs have a crucial function in the occurrence and development of cancer $(21,22)$. Several miRNAs have been revealed as either tumor suppressors or oncogenes in the development of RCC. miR-210, miR-155 and miR-21 are upregulated and highly correlated with patient survival rates in RCC (23). By contrast, miR-708, miR-141 and miR-145 are downregulated in human RCC specimens $(9,24,25)$. The present study revealed that the expression levels of miR-509-3p were downregulated in the 786-O and ACHN cells compared with the normal adjacent RCC tissues. This result was consistent with those of previous sequencing and RT-qPCR analyses in RCC tissues (20). It was hypothesized that miR-509-3p may be important as a tumor suppressor in RCC, therefore, the present study investigated the functional significance of miR-509-3p in RCC and found that miR-509-3p inhibited cell proliferation and migration in 786-O and ACHN RCC cells. Taken together, these results demonstrated the anticancer effect of miR-509-3p, providing sufficient evidence to support the tumor suppressive effect of miR-509-3p in RCC.

The results of the present study highlighted the importance of miR-509-3p as a tumor suppressor in RCC, however, the underlying molecular mechanisms remain to be elucidated. To improve understanding of the tumor suppressive effect of miR-509-3p in renal tumorigenesis, four computational algorithms were used to predict the targets of miR-509-3p, which identified the MAP3K8 gene as a novel target. The target gene and the binding sites of miR-509-3p on the MAP3K8 3'UTR were confirmed using a luciferase reporter assay.

Previous studies have demonstrated that MAP3K8 is a member of the serine/threonine protein kinase family $(17,26,27)$. When overexpressed in cell lines, MAP3K8 activates the extracellular signal-regulated kinase (ERK), JNK and p38 MAPK pathways by inducing the phosphorylation and activation of the respective MAP2K (27). MAP3K8 has been the focus of investigations due to the close association between the expression of MAP3K8 and tumor progression, growth and metastasis. MAP3K8 is important in the promotion of androgen depletion-independent (ADI) prostate cancer progression (15). The overexpression of MAP3K8 correlates with poor prognosis in patients with early-onset CRC cancer (16). The present study revealed that the expression of MAP3K8 was upregulated in RCC cells, suggesting that it acted as an oncogene in RCC.

In the present study, miR-509-3p directly regulated the expression of MAP3K8 in a luciferase reporter assay. The MAP3K 8 oncogene was identified to be negatively regulated by miR-509-3p at the post-transcriptional level through a specific target site within the $3^{\prime}$ UTR of the gene. This suggested that miR-509-3p may exhibit tumor suppressive functions by regulating the oncogene in RCC. Loss of miR-509-3p in RCC may lead to the upregulation of MAP3K8, providing a selective growth and expansion advantage during renal carcinogenesis.

The present study also identified that the overexpression of miR-509-3p suppressed the mRNA and protein expression levels of MAP3K8, and the knockdown of MAP3K8 inhibited the migration and proliferation of the RCC cells. Taken together, these results indicated that miR-509-3p may affect the MAPK signaling pathway is a potential therapeutic target against the MAPK signaling axis in preventing the development and progression of RCC.

In conclusion, the results of the present study demonstrated that miR-509-3p was significantly downregulated in the RCC cell lines and appeared to function as a tumor suppressor in RCC through the regulation of MAP3K8. Therefore, miR-509-3p may be a potential target in the prognosis and treatment of RCC.

\section{Acknowledgements}

This study was supported by the National Natural Science Foundation of China (no. 81101922), the Medical Scientific Research Foundation of Guangdong Province of China (nos. A2012584 and A2013606), the Science and Technology Development Fund Project of Shenzhen (no. CYJ20130402114702124) and the fund of the Guangdong Key Medical Subject.

\section{References}

1. Yamada Y, Hidaka H, Seki N, et al: Tumor-suppressive microRNA-135a inhibits cancer cell proliferation by targeting the c-MYC oncogene in renal cell carcinoma. Cancer Sci 104: 304-312, 2013.

2. Mulders PF, Brouwers AH, Hulsbergen-van der Kaa CA, van Lin EN, Osanto S and de Mulder PH: Guideline 'Renal cell carcinoma'. Ned Tijdschr Geneeskd 152: 376-380, 2008 (in Dutch).

3. Hadoux J, Vignot S and De La Motte Rouge T: Renal cell carcinoma: Focus on safety and efficacy of temsirolimus. Clin Med Insights Oncol 4: 143-154, 2010.

4. Patel C, Ahmed A and Ellsworth P: Renal cell carcinoma: A reappraisal. Urol Nurs 32: 182-190, quiz 191, 2012.

5. Yu ZH, Zhang Q, Wang YD, et al: Overexpression of cyclooxygenase-1 correlates with poor prognosis in renal cell carcinoma. Asian Pac J Cancer Prev 14: 3729-3734, 2013. 
6. Janzen NK, Kim HL, Figlin RA and Belldegrun AS: Surveillance after radical or partial nephrectomy for localized renal cell carcinoma and management of recurrent disease. Urol Clin North Am 30: 843-852, 2003.

7. Bouyssou JM, Manier S, Huynh D, Issa S, Roccaro AM and Ghobrial IM: Regulation of microRNAs in cancer metastasis. Biochim Biophys Acta 1845: 255-265, 2014.

8. Xi Y: MicroRNA: A new player for cancer chemoprevention. J Integr Oncol 2: 105, 2013.

9. Lu R, Ji Z, Li X, Zhai Q, Zhao C, Jiang Z, Zhang S, Nie L and Yu Z: miR-145 functions as tumor suppressor and targets two oncogenes, ANGPT2 and NEDD9, in renal cell carcinoma. J Cancer Res Clin Oncol 140: 387-397, 2014

10. Yu Z, Ni L, Chen D, et al: Identification of miR-7 as an oncogene in renal cell carcinoma. J Mol Histol 44: 669-677, 2013.

11. Peng H,Luo J,Hao H,Hu J,Xie SK, Ren D and Rao B: MicroRNA-100 regulates SW620 colorectal cancer cell proliferation and invasion by targeting RAP1B. Oncol Rep 31: 2055-2062, 2014.

12. Xie J, Chen M, Zhou J, Mo MS, Zhu LH, Liu YP, Gui QJ, Zhang L and Li GQ: miR-7 inhibits the invasion and metastasis of gastric cancer cells by suppressing epidermal growth factor receptor expression. Oncol Rep 31: 1715-1722, 2014.

13. Salmeron A, Ahmad TB, Carlile GW, Pappin D, Narsimhan RP and Ley SC: Activation of MEK-1 and SEK-1 by Tpl-2 proto-oncoprotein, a novel MAP kinase kinase kinase. EMBO J 15: 817-826, 1996.

14. Krcova Z, Ehrmann J, Krejci V, Eliopoulos A and Kolar Z: Tpl-2/Cot and COX-2 in breast cancer. Biomed Pap Med Fac Univ Palacky Olomouc Czech Repub 152: 21-25, 2008.

15. Jeong JH, Bhatia A, Toth Z, Oh S, Inn KS, Liao CP, Roy-Burman P Melamed J, Coetzee GA and Jung JU: TPL2/COT/MAP3K8 (TPL2) activation promotes androgen depletion-independent (ADI) prostate cancer growth. PLoS One 6: e16205, 2011.

16. Aparecida Alves C, Silva ID, Villanova FE, Nicolau SM, Custódio MA, Bortoletto C and Gonçalves WJ: Differential gene expression profile reveals overexpression of MAP3K8 in invasive endometrioid carcinoma. Eur J Gynaecol Oncol 27: 589-593, 2006.

17. Tunca B, Tezcan G, Cecener G, et al: Overexpression of CK20, MAP3K8 and EIF5A correlates with poor prognosis in early-onset colorectal cancer patients. J Cancer Res Clin Oncol 139: 691-702, 2013.
18. Martínez-Salamanca JI, Huang WC, Millan I, et al: Prognostic impact of the 2009 UICC/AJCC TNM staging system for renal cell carcinoma with venous extension. Eur Urol 59: 120-127, 2011. Rini BI, Campbell SC and Escudier B: Renal cell carcinoma. Lancet 373: 1119-1132, 2009.

19. Rini BI, Campbell SC and Escudier B: Renal cell carcinoma. Lancet 373: 1119-1132, 2009.

20. Zhai Q, Zhou L, Zhao C, Wan J, Yu Z, Guo X, Qin J, Chen J and $\mathrm{Lu} \mathrm{R}$ : Identification of miR-508-3p and miR-509-3p that are associated with cell invasion and migration and involved in the apoptosis of renal cell carcinoma. Biochem Biophys Res Commun 419: 621-626, 2012.

21. Li S, Fu H, Wang Y, Tie Y, Xing R, Zhu J, Sun Z, Wei L and Zheng X: MicroRNA-101 regulates expression of the v-fos FBJ murine osteosarcoma viral oncogene homolog (FOS) oncogene in human hepatocellular carcinoma. Hepatology 49: 1194-1202, 2009.

22. Song T, Zhang X, Wang C, Wu Y, Cai W, Gao J and Hong B: MiR-138 suppresses expression of hypoxia-inducible factor $1 \alpha$ (HIF-1 $\alpha$ ) in clear cell renal cell carcinoma $786-O$ cells. Asian Pac J Cancer Prev 12: 1307-1311, 2011.

23. Neal CS, Michael MZ, Rawlings LH, Van der Hoek MB and Gleadle JM: The VHL-dependent regulation of microRNAs in renal cancer. BMC Med 8: 64, 2010.

24. Saini S, Yamamura S, Majid S, Shahryari V, Hirata H, Tanaka Y and Dahiya R: MicroRNA-708 induces apoptosis and suppresses tumorigenicity in renal cancer cells. Cancer Res 71: 6208-6219, 2011.

25. Yu XY, Zhang Z, Liu J, Zhan B and Kong CZ: MicroRNA-141 is downregulated in human renal cell carcinoma and regulates cell survival by targeting CDC25B. Onco Targets Ther 6: 349-354, 2013.

26. Johannessen CM, Boehm JS, Kim SY, et al: COT drives resistance to RAF inhibition through MAP kinase pathway reactivation. Nature 468: 968-972, 2010.

27. Kim K, Kim G, Kim JY, Yun HJ, Lim SC and Choi HS: Interleukin-22 promotes epithelial cell transformation and breast tumorigenesis via MAP3K8 activation. Carcinogenesis 35: 1352-1361, 2014. 